Regular paper for J. Biochem. : Biochemistry (Protein structure)

Closed Complex of the D-3-Hydroxybutyrate Dehydrogenase Induced by an Enantiomeric

\title{
Competitive Inhibitor
}

Kanako Nakashima, Kiyoshi Ito*, Yoshitaka Nakajima, Ryuji Yamazawa, Syunsuke Miyakawa, and

\section{Tadashi Yoshimoto}

Department of Molecular Medicinal Sciences, Graduate School of Biomedical Sciences, Nagasaki

University, 1-14 Bunkyo-machi, Nagasaki 852-8521, Japan

Running title: Closed Structure of the D-3-Hydroxybutyrate Dehydrogenase

*Corresponding Author: Kiyoshi Ito, E-mail: k-ito@nagasaki-u.ac.jp, Tel: +81-95-819-2436, Fax:

$+81-95-819-2478$

Abbreviations: 3-HB, 3-hydroxybutyrate; HBDH, D-3-hydroxybutyrate dehydrogenase; HSDH, hydroxysteroid dehydrogenase; rms, root mean square; SDR, short-chain dehydrogenase/reductase. 


\section{SUMMARY}

D-3-hydroxybutyrate dehydrogenase (HBDH) from Pseudomonas fragi showed a strict stereospecificity to the D-enantiomer of 3-hydroxybutyrate (D-3-HB) as a substrate. The L-enantiomer acts as a competitive inhibitor, with a $K_{\mathrm{i}}$ value comparable to the $K_{\mathrm{m}}$ value for D-3-HB.

We have determined the crystal structures of the ternary complex of HBDH-NAD ${ }^{+}-\mathrm{L}-3-\mathrm{HB}$ and the binary complex of HBDH-NAD ${ }^{+}$. The former structure showed a so-called closed-form conformation, which is considered an active form for catalysis, while the latter stayed mostly in a open-form conformation. The determined structures along with the site-directed mutagenesis confirmed the substrate recognition mechanism that we proposed previously.

The hydrogen bonding interaction between Gln196, located in the moving helix, and the carboxyl group of the substrate/inhibitor is important for the stable ternary complex formation. Finally, the crystal structures of the Thr190 mutants, T190S and T190A, indicate that the Thr190 is a key residue for the open-closed conformational change. T190S retained $37 \%$ of the activity. In T190A, however, the activity decreased to $0.1 \%$ that of the wild-type enzyme. Fixing the position of 
the hydroxyl group of Thr190 to form hydrogen bonds to the pyrophosphate moiety and the carboxamide of $\mathrm{NAD}^{+}$seems to be a significant factor for the open-closed conformational change.

Key words: hydroxybutyrate dehydrogenase; short-chain dehydrogenase/reductase family; crystal structure; conformational change; substrate recognition 


\section{INTRODUCTION}

D-3-hydroxybutyrate dehydrogenase (HBDH: EC 1.1.1.30) is an enzyme that reversibly oxidizes

3-hydroxybutyrate to acetoacetate using $\mathrm{NAD}^{+}$as a coenzyme. Mammals metabolize acetyl-CoA, which is formed during oxidation of fatty acids, by either of two pathways. One pathway is the citric acid cycle, while the other leads to acetoacetate and D-3-hydroxybutyrate, which, together with acetone, are collectively called ketone bodies. HBDH, a mitochondrial enzyme, reversibly reduces the free acetoacetate so produced into D-3-hydroxybutyrate (1).

One of the severe complications of diabetes mellitus is diabetic ketoacidosis (DKA). DKA is caused by an increase of ketone bodies. It is important to monitor ketone bodies in the blood and urine of patients with diabetes mellitus (1). Mammalian HBDH is a mitochondrial enzyme that specifically requires phosphatidylcholine for activity (2). On the other hand, bacterial HBDH is a cytosolic enzyme and does not require phospholipids for its activity (3). Microbial enzymes have been used for the diagnostic analysis of ketone bodies for diabetes mellitus (4). 
HBDH belongs to the SDR family (5). More than 30000 primary sequences are deposited in the sequence databases, and approximately 300 crystal structures are available. The SDR family enzymes act on a great variety of compounds and are divided into several EC classes, from oxidoreductases to isomerases. Moreover, within the subgroup of oxidoreductases to which most of the enzymes belong, diverse substrate specificities are found to act on a range of compounds, from steroids to aliphatic alcohols. The diversity of the SDR family might be in the same range as that of the CYP P450 family (6). The amino acid sequence identity among SDRs is relatively low, roughly $15-30 \%$. However, the three-dimensional structures of the SDR family enzymes have architectures that are quite similar to each other, except for the substrate-binding loop region (7). Based on the functional assignments of coenzyme binding motifs, SDR family was divided into 5 subfamilies, called classical, extended, intermediate, divergent, and complex (8). The catalytic tetrad of Asn-Ser-Tyr-Lys is conserved in most of oxidoreductases in the family (9). Since some particular SDR members could be drug targets and some could be useful as enzyme reagents in organic synthesis or clinical chemistry, it is very important to understand the strategies used to recognize SDR substrates. 
One of the striking features of SDR enzymes, mainly for the classical type, is a large conformational change upon substrate binding $(5,7,10,11)$. The most variable region of the substrate-binding loop moves to cover the substrate for catalysis. We have solved the crystal structures of the complex of HBDH from Pseudomonas fragi (PfHBDH) with a competitive inhibitor and with the inhibitor and $\mathrm{NAD}^{+}$(5). While the structure of the PfHBDH-inhibitor complex showed an open-form conformation, that of the PfHBDH-NAD ${ }^{+}$-inhibitor complex showed a closed-form conformation. In the latter complex, however, the structure of the substrate-binding loop was disordered. Recently, three crystal structures closely related to the PfHBDH have been reported. DHRS6 is a human cytosolic type II HBDH having high $K_{\mathrm{m}}$ and low $k_{\text {cat }}$ values (12). Two bacterial HBDHs, one from Pseudomonas putida (PpHBDH) and the other from Alcaligenes faecalis (AfHBDH), were also reported $(13,14)$. The structures of bacterial HBDHs contained a closed conformation.

P. fragi HBDH catalyzed the dehydrogenation of only the D-enantiomer of 3-hydroxybutyrate. Our previous study indicated that this stringent substrate specificity was achieved through the recognition of the 3-methyl and carboxyl groups of the substrate. These two major interactions fixed both ends of the 
substrate carbon chain. In the SDR family, a variable region known as a substrate-binding loop moves to cover the substrate upon substrate binding. As a result, only the $3 R$-configuration of the hydroxyl group is thought to orient to the catalytic tetrad of N114-S142-Y155-K159 of the enzyme, thus allowing the hydride transfer to occur. The hydrophobic pocket to accommodate the 3-methyl group is composed of Ala143, His144, Gly186, Trp187, and Trp257. The carboxyl group is recognized through interactions with Gln94, His144, and Lys152 (5).

In this study, we constructed various site-directed mutants of PfHBDH to confirm the substrate recognition mechanism that we proposed in our previous work. Specific mutations were also introduced to Thr190 and Leu215, which are thought to function as hinges by which the substrate-binding loop moves to cover the bound substrate. Finally, we determined the structure of a ternary complex of $\mathrm{HBDH}, \mathrm{NAD}^{+}$, and L-3-hydroxybutyrate as a closed form. These results allowed us to describe the detailed mechanisms underlying substrate recognition and conformational change.

\section{MATERIALS AND METHODS}




\section{Materials}

Restriction endonucleases and other DNA modification enzymes were purchased from TaKaRa

Shuzo Co. The oligonucleotide primers were synthesized by Genenet. The BigDye terminator cycle sequencing kit and other reagents used for sequencing were obtained from Applied Biosystems. 3-D(-)(3R)-hydroxybutyric acid sodium salt, 3-L(+)(3S)-hydroxybutyric acid sodium salt, and D-threonine were purchased from Sigma-Aldrich. Phenazine methosulfate (PMS), nitro blue tetrazolium (NBT), and Triton X-100 are products of Nacalai Tesque. $\mathrm{NAD}^{+}$was from Kojin Kagaku. Polyethylene glycol, which was used for crystallization and had a mean molecular weight of 3000 (PEG3000), was purchased from Hampton Research. L-threonine, L-serine, and all other chemicals were of the highest grade available from Nacalai Tesque or Wako Pure Chemical Industries.

\section{Bacterial strains and plasmids}

E. coli DH5a ( $\mathrm{F}^{-}, \phi 80 \mathrm{~d} l a c Z \Delta \mathrm{M} 15, \Delta($ lacZYA-argF)U169, recA1, endA1, gyrA96, thi-1, supE44, relA1, hsdR17(r-, m+), deoR, phoA) and XL1Blue (Stratagene) were used for DNA manipulation and 
protein expression. pGEM-T-easy (Promega) was used for cloning PCR products, and pQE30 (Qiagen) and pKK223-3 (Pharmacia) were used for protein expression.

\section{Site-directed mutagenesis}

Specific mutations were introduced into the gene by a PCR-based megaprimer method, which consisted of two rounds of PCR amplification (15). In the first round, one of the flanking primers and the internal mutagenic primer were used to generate a megaprimer. In the second round, another flanking primer and the megaprimer were used to generate the DNA fragment with the desired mutation. Table 1 summarizes the mutagenic and flanking primers along with restriction enzymes used to ligate the DNA fragments containing the desired mutations into pHBDH11 or pHBDHX (pQE30). PCR conditions consisted of 25 cycles of denaturation at $95^{\circ} \mathrm{C}$ for $30 \mathrm{sec}$, annealing at $57^{\circ} \mathrm{C}$ for $30 \mathrm{sec}$, and extension at $72^{\circ} \mathrm{C}$ for $30 \mathrm{sec}$, with a final extension at $72^{\circ} \mathrm{C}$ for $7 \mathrm{~min}$. Amplified DNA fragments from the first round of PCR were purified by the QIAEX II kit (Qiagen) from agarose gel and used as megaprimers for the second 
round of PCR. The condition consisted of 25 cycles of denaturation at $95^{\circ} \mathrm{C}$ for $30 \mathrm{sec}$, annealing at $40^{\circ} \mathrm{C}$ for $60 \mathrm{sec}$, and extension at $72^{\circ} \mathrm{C}$ for $60 \mathrm{sec}$.

\section{Purification of the recombinant enzymes}

The wild-type, Y155F, T190A, T190S, and T190C mutant enzymes were expressed in E. coli

XL1Blue harboring pHBDH11 and its derivatives containing the specific mutations. The wild-type HBDH was purified as described previously (5). Four kinds of mutants (Y155F, T190A, T190S, and T190C) were purified by essentially the same procedure as that used for the wild-type enzyme. The wild-type HBDH containing an $\mathrm{N}$-terminal histidine-tag $\left(\mathrm{H}_{6}-\mathrm{HBDH}\right)$ and the other mutant enzymes, each containing an $\mathrm{N}$-terminal histidine tag, were expressed in E. coli XL1Blue using pQE30 as a vector. Briefly, the transformants were cultured in 2 liters of $\mathrm{LB}$ containing $50 \mathrm{mg} / 1$ ampicillin at $37^{\circ} \mathrm{C}$ overnight. Cells were harvested by centrifugation at $10,000 \mathrm{x}$ g for $15 \mathrm{~min}$ at $4^{\circ} \mathrm{C}$ and resuspended in $300 \mathrm{ml}$ of $20 \mathrm{mM}$ Tris- $\mathrm{HCl}$ (pH 7.5). Cell lysates were prepared by repeated sonication in an ice bath using an Ultrasonic Disruptor UD200 (TOMY, Japan) followed by centrifugation at $15,000 \mathrm{x}$ g for $20 \mathrm{~min}$. The crude lysates were 
directly applied onto a Ni-affinity column (GE Healthcare) and the column was washed with $500 \mathrm{ml}$ of 20 $\mathrm{mM}$ Tris- $\mathrm{HCl}$ buffer ( $\mathrm{pH} 7.5$ ) containing $0.5 \mathrm{M} \mathrm{NaCl}$ and $0.1 \mathrm{M}$ imidazole. The adsorbed enzymes were eluted with $20 \mathrm{mM}$ Tris- $\mathrm{HCl}$ buffer $(\mathrm{pH} 7.5)$ containing $0.5 \mathrm{M} \mathrm{NaCl}$ and $0.3 \mathrm{M}$ imidazole, and the fractions showing significant absorbance at $280 \mathrm{~nm}$ were collected. Enzyme proteins in the collected fractions were precipitated by ammonium sulfate at $80 \%$ saturation. After centrifugation at $15,000 \times \mathrm{g}$ for $20 \mathrm{~min}$, the enzymes were resuspended in a small volume of $20 \mathrm{mM}$ Tris-HCl buffer $(\mathrm{pH} 7.5)$ and dialyzed against the same buffer. The dialysates were applied to a DEAE-Toyopearl 650C column and washed with the same buffer. The enzymes were eluted using a linear gradient from 0 to $0.5 \mathrm{M} \mathrm{NaCl}$ in the same buffer. Active fractions were collected and achieved $80 \%$ saturation of ammonium sulfate. After centrifugation at 15,000 $\mathrm{x} \mathrm{g}$ for $20 \mathrm{~min}$, the enzymes were resuspended in a small volume of $10 \mathrm{mM}$ Tris- $\mathrm{HCl}$ buffer (pH 7.5) and dialyzed against the same buffer. The purified enzymes were lyophilized and stored at $-20^{\circ} \mathrm{C}$ until use. SDS-PAGE analysis showed that all the purified enzymes were apparently homogeneous. The molecular mass of the wild-type enzyme was $27 \mathrm{kDa}$, and the enzymes having an N-terminal histidine tag were detected as slightly larger bands of $28 \mathrm{kDa}$. 


\section{Kinetic analysis}

The kinetic parameters were determined by the Lineweaver-Burk plot using the initial rate data

measured as described previously (5). While $K_{\mathrm{m}}$ for D-3-HB was determined with various concentrations of D-3-HB $(0.1$ to $100 \mathrm{mM})$ in the presence of $2 \mathrm{mM} \mathrm{NAD}^{+}, K_{\mathrm{m}}$ for $\mathrm{NAD}^{+}$was determined with various concentrations of $\mathrm{NAD}^{+}(0.1$ to $2 \mathrm{mM})$ in the presence of $25 \mathrm{mM}$ and $100 \mathrm{mM}$-3-HB for the wild-type and mutant enzymes, respectively. Similarly, acetoacetate reduction was monitored by measuring absorbance at $340 \mathrm{~nm} . K_{\mathrm{m}}$ for acetoacetate was determined with various concentrations of acetoacetate $(0.2$ to $50 \mathrm{mM})$ in the presence of $0.25 \mathrm{mM} \mathrm{NADH} . K_{\mathrm{m}}$ for NADH was determined with various concentrations of NADH ( 0.01 to $0.25 \mathrm{mM}$ ) in the presence of $25 \mathrm{mM}$ acetoacetate.

\section{Crystallization and data collection}

Crystallization was carried out at $293 \mathrm{~K}$ with the hanging-drop vapor-diffusion method using 15-17\% (w/v) PEG8000, $100 \mathrm{mM} \mathrm{MgCl}$, and $100 \mathrm{mM}$ HEPES-Na buffer $\mathrm{pH} 7.5$ as a reservoir solution. 
The binary complex of the wild-type enzyme with $\mathrm{NAD}^{+}$was crystallized by equilibrating a droplet of $2 \mu 1$ solution $\left(39.8 \mathrm{mg} / \mathrm{ml}\right.$ protein solution containing $4 \mathrm{mM} \mathrm{NAD}^{+}$mixed with the same amount of a reservoir solution) against $500 \mu 1$ of the reservoir solution. Crystallization of the ternary complex was carried out with the same method as the binary complex using $37.7 \mathrm{mg} / \mathrm{ml}$ protein solution containing $8 \mathrm{mM} \mathrm{NAD}^{+}$and 25 mM L-3-HB. The crystals of mutant enzymes, T190S and T190A mutants, were obtained by the same method as the wild-type enzyme using $30.0 \mathrm{mg} / \mathrm{ml}$ protein solution containing $24 \mathrm{mM} \mathrm{NAD}^{+}$and $50 \mathrm{mM}$ L-3-HB. After 1 week, column crystals grew to average dimensions of $0.1 \times 0.05 \times 0.5 \mathrm{~mm}$.

The data were collected at $100 \mathrm{~K}$ using a wavelength of $1.0000 \AA$ from the synchrotron radiation source at the Photon Factory BL17 station (Tsukuba, Japan) with an ADSC Quantum Q270 CCD detector system and the Spring-8 BL38B1 station (Hyogo, Japan) with a RIGAKU Jupiter210 CCD detector system. To collect data under cryogenic conditions, crystals were soaked for a few seconds in a solution containing $30 \%$ (v/v) glycerol, $18 \%$ (w/v) PEG 8000, $200 \mathrm{mM} \mathrm{MgCl}_{2}$, and $100 \mathrm{mM}$ HEPES-Na buffer $\mathrm{pH}$ 7.5. Crystals were mounted in a nylon loop and flash-cooled in a nitrogen stream at $100 \mathrm{~K}$. All data sets were processed and scaled using HKL2000 (Table 2) (16). 


\section{Structure determination and refinement}

We have reported the structures of HBDH from $P$. fragi complexed with cacodylate (PDB code: $1 \mathrm{WMB}$, open-form) and of NAD ${ }^{+}$and cacodylate (PDB code: $\left.1 \mathrm{X} 1 \mathrm{~T}\right)(5,17)$. Those crystals belong to the space groups $P 22_{1}{ }_{1} 2$ and $I 222$, respectively. Meanwhile, the crystals obtained under new condition without cacodylate belong to the space group $P 2{ }_{1}{ }_{2}{ }_{1}{ }_{1}$ with the following cell constants: $a=73.2, b=106.8$, and $c=163.2 \AA$ on the binary complex. One tetramer is found in an asymmetric unit, and $59 \%$ of the crystal volume is occupied by solvent. The structure of the binary complex was determined by the molecular replacement method with a tetramer constructed from $1 \mathrm{WMB}$ as an initial model using the program Molrep (18) in the CCP4 program suite (19). The structure of the binary complex was refined by simulated annealing and energy minimization using the CNS program (20). The structure was scrutinized by inspecting the composite omit map using the program Coot (21). A difference map obviously displayed a residual peak from the $\mathrm{NAD}^{+}$at the active site in each subunit. After several rounds of refinement using the program Refmac5 (22) in the CCP4 program suite (19) and manual rebuilding, water molecules were 
picked up on the basis of the peak height and the distance criteria from the difference map using the Coot program (21). On a difference map, one peak was found to be assigned a glycerol molecule by considering the shape, size, peak height, and possible interactions of this peak with neighboring amino acid residues; two high peaks were found at positions corresponding to center of symmetry of the dimmer. Since two peaks were located at the center of face-to-face C-terminal carboxyl groups, these were assigned as magnesium ions six-coordinated to two carboxyl groups and four water molecules. Further model building and refinement cycles resulted in an $R_{\text {factor }}$ of $20.0 \%$ and $R_{\text {free }}$ of $23.5 \%$, using 88463 reflections from 20 to $1.95 \AA$ resolution (Table 2). The structure of the ternary complex was refined in the same way as that of the binary complex. At each active site, the difference Fourier map showed the residual peaks corresponding to $\mathrm{NAD}^{+}$and L-3-HB. From the result of model building and refinement cycles, the $R_{\text {factor }}$ and $R_{\text {free }}$ values were reduced to $19.8 \%$ and $22.8 \%$, respectively, calculated for 115106 reflections from 20 to $1.80 \AA$ R resolution (Table 2). The structures of two mutants were refined in the same way. In the T190S mutant, an $\mathrm{NAD}^{+}$was found in three subunits of the tetramer, and an L-3-HB was bound to one of them. In the T190A mutants, an $\mathrm{NAD}^{+}$was found in three subunits of the tetramer. After several rounds of model building and refinement, 
the $R_{\text {factor }}$ and $R_{\text {free }}$ values on the T190S and $190 \mathrm{~A}$ mutants were reduced to $22.7 \%$ and $26.6 \%$ using 54951 reflections from 20 to $2.3 \AA$ resolution, and $23.8 \%$ and $26.8 \%$ using 81824 reflection from 20 to $2.0 \AA$ resolution, respectively (Table 2).

\section{PDB accession codes}

The atomic coordinates and structure factors for wild-type enzyme complexed with $\mathrm{NAD}^{+}$(the binary complex), wild-type enzyme complexed with $\mathrm{NAD}^{+}$and L-3-HB (the ternary complex), T190S mutant, and T190A mutant (PDB code: 2ZTV, 2ZTL, 2ZTM, and 2ZTU) have been deposited in the Worldwide Protein Data Bank (wwPDB; http://www.wwpdb.org) and in the Protein Data Bank Japan at the Institute for Protein Research, Osaka University (PDBj; http://www.pdbj.org/).

\section{RESULTS}

\section{Expression and purification of the various $\mathrm{HBDH}$ mutants}


Among the residues involved in the substrate specificity, 6 strictly conserved residues were chosen for site-directed mutagenesis. To simplify the purification of the wild-type and mutant HBDHs, a 6 $\mathrm{x}$ histidine tag was introduced to their N-termini. All the mutants were well expressed to similar levels, and the expressed enzymes were efficiently purified by metal chelating affinity chromatography followed by ion-exchange chromatography on a Toyopearl SuperQ-650C column. A comparison of the kinetic parameters between the wild-type HBDH with the histidine tag and that without showed that both HBDHs have almost the same enzymatic characteristics. This indicated that the $\mathrm{N}$-terminal histidine tag did not interfere with enzyme activity. This agrees well with the structure determined previously, in which the $\mathrm{N}$-terminus of the enzyme is located far from the active site and is not involved in subunit interactions. The kinetic parameters are summarized in Table 3.

\section{Recognition of the carboxyl group}

Gln94, His144, Lys152, and Gln196 were replaced by alanine. Large decreases in activity were

observed for all the alanine mutants. Lys 152 was found to be essential, since no activity was detected for the 
K152A mutant. The catalytic efficiencies $\left(k_{\text {cat }} / K_{\mathrm{m}}\right)$ of Q94A and H144A were $1.4 \%$ and $0.2 \%$ of the activity of wild-type $\mathrm{HBDH}$, suggesting these residues contributed significantly to the carboxyl recognition. Arginine substitution for lysine showed a significant level of activity. The $K_{\mathrm{m}}$ value was 40 -fold that of the wild type, while the $k_{\text {cat }}$ value was approximately $40 \%$ that of the wild type, indicating not only the importance of a positive charge to interact with the carboxyl group of the substrate but also that the positive charge was not directly involved in the catalytic reaction.

\section{Recognition of the 3-methyl group}

Trp187 and Trp257 were mutated. Very little activity was detected for the W187A mutant, and none was found for the W257A mutant. This is in good agreement with the finding that Trp257 was strictly conserved among various HBDH sequences. The residue at position 187 was often a tryptophan, but tyrosine and phenylalanine could be found in some bacterial HBDH sequences. We also made tyrosine and phenylalanine substitutions for these Trp residues. W187F and W187Y mutants showed significant activity levels--65\% and $41 \%$ that of the wild-type enzyme, respectively. W257F and W257Y showed only faint 
levels-- $2 \%$ and $1 \%$ of that of the wild-type enzyme, respectively. The decrease in W187A activity was mainly due to the 100 -fold increase in $K_{\mathrm{m}}$ value, suggesting that a bulky hydrophobic residue is required at this position to accommodate the 3-methyl group of the substrate. Tyrosine and phenylalanine may be sufficient in this regard. However, as the decreased $k_{\text {cat }}$ values for W187F and W187Y suggest, tryptophan was preferable for fixing the substrate to a better orientation. It can be concluded that Trp 257 is essential for enzyme activity. In addition, a W187T mutant was constructed to investigate the very low activity of the human type $2 \mathrm{HBDH}$ (12). The overall structures of $P$. fragi $\mathrm{HBDH}$ and human type $2 \mathrm{HBDH}$ are very similar despite their low sequence identity (18\%). Most of the functional residues important for substrate recognition and enzymatic activity are conserved except for Lys152 and Trp187, which are Arg144 and Thr179, respectively, in human type $2 \mathrm{HBDH}$. W187T showed faint activity. Since the K152R mutant retained a significant level of activity, Thr179 in the human type 2 HBDH may be the major cause of its low activity, although the functional relevance of Thr179 is unclear.

\section{Interaction between substrate entry loop and the LHB}


In our previous model, Pro191-Glu214, which corresponded to the substrate binding loop of the closed form of $P$. fragi HBDH, were missing. This region shows a limited sequence identity and is very important for substrate recognition in individual SDR enzymes. For mutagenesis, we chose Gln196, which is strictly conserved among HBDH sequences. The $k_{\mathrm{cat}} / K_{\mathrm{m}}$ value of the Q196A mutant was only $0.6 \%$ that of the wild type, and substitution by glutamate and aspartate also substantially reduced activity. As described later, Gln196 formed a hydrogen bond with the carboxyl group of the substrate.

\section{Importance of the residues functioning as hinges}

Comparison of the open- and closed-form structures clearly showed the conformational change

of the two key residues, Thr190 and Leu215, to enable the hinge movement of the substrate-binding loop as hinges (5). Thr190 was replaced by serine, alanine, and cysteine, and Leu215 was replaced by valine and alanine. These mutants were expressed without histidine tags and purified to homogeneity for both kinetic and crystallographic analyses. Since the hydroxyl group of Thr190 was considered to interact with a pyrophosphate and a carboxamide of $\mathrm{NAD}^{+}$molecules, we determined the kinetic parameters for $\mathbf{D}-3-\mathrm{HB}$, 
acetoacetate, $\mathrm{NAD}^{+}$, and NADH (Table 4). As expected, significant activity was observed for T190S. The mutations increased the $K_{\mathrm{m}}$ values 2- to 4 -fold but did not decrease the $k_{\mathrm{cat}}$ values, which indeed even slightly increased. In the T190A mutant, $K_{\mathrm{m}}$ and $k_{\text {cat }}$ values showed a much larger increase and decrease, respectively. The activity was decreased also in T190C. We tried to measure the activity of the T190C mutant at $\mathrm{pHs}$ below the optimum of 8.5 . The activity ratio of T190C and the wild type were similar through the $\mathrm{pH}$ range tested, indicating that the sulfhydryl group cannot substitute for the hydroxyl group of Thr190. Interestingly, similar changes in kinetic parameters were observed for Leu215 mutants. In the L215V mutant, $K_{\mathrm{m}}$ values increased 3.5- and 4.3 -fold and the $k_{\text {cat }}$ values were $73 \%$ to $118 \%$ those of the wild-type toward D-3-HB and acetoacetate, respectively. The mutation did not significantly change $K_{\mathrm{m}}$ and $k_{\text {cat }}$ toward $\mathrm{NAD}^{+}$and NADH. In the L215A mutant, both $K_{\mathrm{m}}$ and $k_{\text {cat }}$ values were largely affected and the catalytic efficiency $\left(k_{\mathrm{cat}} / K_{\mathrm{m}}\right)$ was less than $3 \%$ that of the wild-type enzyme.

\section{Qualities of the structures}


The final model of the binary complex contains a homotetramer composed of $260,246,255$, and 253 amino acid residues (subunits $\mathrm{A}, \mathrm{B}, \mathrm{C}$, and $\mathrm{D}$, respectively), four $\mathrm{NAD}^{+}$, two magnesium ions, a glycerol, and 578 water molecules, with an $R$-factor of $20.0 \%$ at $1.95 \AA$ resolution. The model lacks 26 residues (Glu196-Glu209, Leu200-Asn204, and Ala199-Gly205 in chains B, C, and D, respectively), and the average thermal factors of the main chain atoms at subunits A, B, C, and D are 34.6, 46.3, 31.6, and 33.7 $\AA^{2}$, respectively. Analysis of the stereochemistry with PROCHECK (23) showed that all of the main chain atoms fall within the generously allowed region of the Ramachandran plot, with 784 residues in the most favored region, 73 residues in the additionally allowed region, and 2 residues in the generously allowed region. The ternary complex contains a homotetramer, each of whose subunits contains 260 amino acid residues, four $\mathrm{NAD}^{+}$, four $\mathrm{L}-3-\mathrm{HB}$, two magnesium ions, and 836 water molecules, with an $R$-factor of $19.8 \%$ at $1.80 \AA$ resolution. The average thermal factor of main chain atoms at subunits A, B, C and D are 23.9, 37.7, 23.9, and $24.0 \AA^{2}$, respectively. Analysis of the stereochemistry with PROCHECK showed that 815,70 , and 3 residues fall within the most favored, the additionally allowed, and the generously allowed regions of the Ramachandran plot, respectively. The T190S mutant contains a homotetramer composed of 
255, 224, 250, and 247 residues (subunits A, B, C and D, respectively), three $\mathrm{NAD}^{+}$, an L-3-HB, two magnesium ions, and 408 water molecules, with an $R$-factor of $22.7 \%$ at $2.30 \AA$ resolution. The model lacks 55 residues (Gly37-Ala47 and Pro191-Ala211 in subunit B, Asn204-Arg213 in subunit C, and Leu200-Ala212 in subunit D). The average thermal factor of main chain atoms at subunits A, B, C, and D are 41.7, 57.5, 31.6, and $37.0 \AA^{2}$, respectively. Analysis of the stereochemistry with PROCHECK showed that 716,106 , and 2 residues fall within the most favored, the additionally allowed, and the generously allowed regions of the Ramachandran plot, respectively. The T190A mutant contains a homotetramer composed of 245, 214, 242, and 241 residues (subunits A, B, C, and D, respectively), three $\mathrm{NAD}^{+}$, two magnesium ions, and 633 water molecules, with an $R$-factor of $23.8 \%$ at $2.0 \AA$ resolution. The model lacks 108 residues (Ala190-Glu214 in subunit A, Gly37-Val57 and Arg189-Arg213 in subunit B, Ala190-Asp207 in subunit C, and Ala190-Glu208 in subunit D). The average thermal factors of main chain atoms at subunits A, B, C, and D are 31.3, 47.5, 31.7, and $28.5 \AA^{2}$, respectively. Analysis of the stereochemistry with PROCHECK showed that 690,83 , and 2 residues fall within the most favored, the additionally allowed, and the generously allowed regions of Ramachandran plot, respectively. Refinement statistics is summarized in 
Table 2. Structure diagrams were drawn with the Molscript (24) POVscript ${ }^{+}$(25), and Raster3D (26) programs.

\section{Overall and subunit structures}

The structure of the ternary complex with $\mathrm{NAD}^{+}$and L-3-HB was determined. As shown in Figure 1a, four subunits, except for the substrate-binding region in the ternary complex, were superimposed using a least-squares method at $\mathrm{C} \alpha$ positions on the open-formed structure of $1 \mathrm{WMB}$ with rms deviation values of $0.28-0.35 \AA$. The structure of the substrate-binding region of the ternary complex was significantly different from that of the open form and was considered a closed form of the HBDH. In fact, the conformation of subunit A was similar to that of $\mathrm{HBDH}$ complexed with $\mathrm{NAD}^{+}$and acetate anion from Alcaligenes faecalis (PDB code: 2ZEA) (14), and two structures can be superimposed with an rms deviation value of $0.48 \AA$.

The subunit structures of the binary complex with $\mathrm{NAD}^{+}$were compared with those of the open and closed forms, as shown in Figure 1b. Four subunits, except for the substrate-binding regions, were 
superimposed on the open form with rms deviation values of $0.27-0.37 \AA$. The conformation of the substrate binding regions at subunit A apparently corresponded to the closed form, and those of subunits B, $\mathrm{C}$, and $\mathrm{D}$ were found to exist as open forms, although a part of the substrate binding region was disordered. Each subunit structure of the T190S and T190A mutants was superimposed using a least-squares method on the open-form structure with an rms deviation value of approximately $0.30 \AA$. At every active site of the T190S mutant except for subunit B, a Fourier peak was identified as an NAD ${ }^{+}$. Only one peak was obviously observed at the active site of subunit A, corresponding to L-3-HB. The same peak was obscure in the other subunits. From the comparison of subunit structures shown in Figure 1c, the conformation of subunit A clearly adopted the closed form, and the others were also considered to have the closed form. In the T190A mutant, a Fourier peak corresponding to $\mathrm{NAD}^{+}$was observed at the active site except for that of subunit B. Although the crystallization solution contained the same concentration of L-3-HB as used for T190S, Fourier peaks from L-3-HB were not found at any of the active site in the subunits. The conformation of the subunits corresponded approximately to the open form shown in Figure 1d. The conformation of Trp187, which is important in recognizing the 3-methyl group of a substrate, 
changes upon substrate binding (5). In the T190A mutant, the conformation of Trp187 in subunits A and C, or that in subunits B and D, mainly had an open or closed form, respectively, indicating that the crystal structure of the T190A mutant contains a mixture of two conformations. Conformations of the individual subunits in various complexes are summarized in Table 5.

\section{Closed conformation of $\mathrm{HBDH}$}

All the subunits of the ternary complex with $\mathrm{NAD}^{+}$and L-3-HB had a closed-form conformation.

A comparison of this with the structure having the open-form conformation revealed that the substrate binding region, Thr190-Leu216, which is composed of two helices and a connecting loop, was rotated approximately $28^{\circ}$ from its position in the open form in order to close the active site (Figure 2). Thr190 and Leu216 were significantly different from the other residues except for Gly in the torsion angle of the main chain between the open and closed forms (Table 6). The variations in the torsion angles $\varphi$ and $\psi$ were $-19.0^{\circ}$ and $7.4^{\circ}$ at $\mathrm{Thr} 190$, and $20.0^{\circ}$ and $-29.7^{\circ}$ at Leu216, respectively. This indicates that the substrate binding region rotated on Thr 190 and Leu216 as center of hinges. As a result of this conformational change, the side 
chain of Thr190 was rotated approximately $37^{\circ}$, and a hydroxyl group of Thr190 formed a hydrogen bond to the pyrophosphate moiety of $\mathrm{NAD}^{+}$with a distance of $2.49 \AA$. In addition, the active site was covered by Leu192 and Val193, which were located on the substrate binding region, at displacements of 5.07 and 3.43 $\AA$, respectively, thus separated from solvent. Trp187 was involved in the recognition of the 3-methyl group of a substrate at $\chi_{2}$ angle variation of $26.3^{\circ}$. The rotation of Trp187 was induced by a displacement of Leu215 with $2.27 \AA$. Leu215 that is located at the hinge region formed a hydrophobic interaction to Trp187.

The L-3-HB is tightly bound to the active site in each subunit, as shown in Figure 3. The mean thermal factors of L-3-HB were 25.4, 38.6, 28.9, and $36.4 \AA^{2}$ in subunits A-D, respectively. The 3-methyl group binds to a hydrophobic pocket consisting of Ala143, His144, Gly186, Trp187, and Trp257. The 3-hydroxyl group forms hydrogen bonds with Ser142 and Tyr155 at distances of 2.45 and $2.54 \AA$, respectively. The carboxyl group is recognized by Gln94, His144, Lys152, and Gln196, to which the distances are $2.97,2.81,2.81$, and $2.68 \AA$, respectively.

\section{DISCUSSION}


We have determined two structures of the HBDH from P. fragi (PDB code: 1WMB, 1X1T) in the presence of a competitive inhibitor, cacodylate (5). The subunit of HBDH is composed of a typical Rossmann fold and the substrate-binding region. The subunit can change its conformation from open to closed form upon substrate binding, as do other members of the SDR family. One of the two structures, 1WMB, is an open form, and a cacodylate anion is bound to the active site. The other is the ternary complex of $\mathrm{HBDH}-\mathrm{NAD}^{+}$-cacodylate. This ternary complex is considered to have a closed form, although its substrate-binding region is disordered. Cacodylate anion is a competitive inhibitor, and the structural difference between cacodylate and $\mathbf{D}-3$-HB causes the disorder in the substrate-binding region, as described previously (5). In the present study, the closed form structure containing the substrate-binding region was determined by using crystals obtained from cacodylate-free buffer in the presence of NAD ${ }^{+}$and L-3-HB. The crystal structure of the ternary complex of HBDH-NAD ${ }^{+}-\mathrm{L}-3-\mathrm{HB}$ instantly confirmed our hypothesis that stringent stereo-specificity was achieved through the recognition of the carboxyl group and the methyl group of the substrate. The substrate molecule fixed at both ends of its carbon chain was covered by the substrate-binding loop. The hydrogen atom at the 3-position of L-3-HB faced away from the 
4-position of the nicotinamide ring in the complex. In the case of D-3-HB, effective hydride transfer can take place. In addition, the structure also explains why the $K_{\mathrm{i}}$ value of L-3-HB was comparable to the $K_{\mathrm{m}}$ value of the $\mathbf{D}-3-\mathrm{HB}$.

In the HBDH-NAD ${ }^{+}-\mathrm{L}-3-\mathrm{HB}$ complex, closed form is observed for all chains $\mathrm{A}, \mathrm{B}, \mathrm{C}$, and $\mathrm{D}$ of the tetramer. The closed form is confirmed from the conformations of Thr190 and Leu216. O3 of the L-3-HB forms hydrogen bonds to the hydroxyl groups of Tyr155 and Ser142. The carboxyl group of the L-3-HB is bound to the side chains of His144, Lys152, Gln94, and Gln196 by hydrogen bonds of lengths $2.97,2.81,2.81$, and $2.68 \AA$, respectively. These residues are strictly conserved among bacterial HBDH sequences, and this binding occurs in almost the same manner as we suggested from the binding mode of cacodylate in our previous paper. The methyl group at the 3-position of the L-3-HB is accommodated by the hydrophobic pocket consisting of Ala143, Trp187, and Trp257, which are also the same residues as we suggested in our previous paper. We confirmed these interactions by site-directed mutagenesis. Our data are consistent with those reported by Feller et al (28). The enzyme recognizes the carboxyl and 3-methyl groups. The $K_{\mathrm{m}}$ for D-3-HB is comparable to the $K_{\mathrm{i}}$ for L-3-HB. If the enzyme accommodates the 3-methyl 
group of two enantiomers tightly and in exactly the same way, it may be difficult to form a hydrogen bonding interaction between the 3-hydroxyl group of the L-3-HB and Tyr155. However, the 3-hydroxyl group forms hydrogen bonds with Ser142 and Tyr155, as described above. There appears to be some allowance between the inhibitor/substrate and the enzyme. In order to bind the real substrate $\mathbf{D}-3-\mathrm{HB}$, the positions of the $\mathrm{C} 2$ and $\mathrm{C} 3$ atoms must be shifted by approximately $1-2 \AA$ from the corresponding positions of the L-3-HB, as shown in Figure 4. A small clearance of the substrate-binding pocket allows the enzyme to bind L-3-HB, and this binding is achieved through the same interactions for $\mathbf{D}-3-\mathrm{HB}$, which includes the recognition of the carboxyl-, 3-methyl-, and 3-hydroxyl groups. As a result, the enantiomeric inhibitor of the substrate can induce the conformational change to the closed form. No D-3-HB was identified in HBDH crystals obtained from the buffer containing $\mathrm{NAD}^{+}$and $\mathbf{D}-3-\mathrm{HB}$, probably because the catalytic reaction proceeded. Then we tried to obtain crystals of the Y155F-NAD ${ }^{+}-\mathbf{D}-3-\mathrm{HB}$ complex. If the enzyme recognizes D-3-HB mainly by its carboxyl and methyl groups, a closed conformation might be formed. No D-3-HB molecule was observed in the crystals of Y155F enzyme made in the presence of $\mathrm{NAD}^{+}$and D-3-HB (data not shown), indicating that Tyr155 is essential not only for catalysis but also for substrate 
binding. The Y155F mutant enzyme exists basically as an open conformation in the complex. Chain A seems to be a closed form, but it is not possible to confirm the conformations of the other chains because they are partially disordered. Although the disorder may be an indication of the $\mathrm{NAD}^{+}$-induced conformational change to the closed structure, a stable ternary complex was not observed. Tyr 155 forms a hydrogen bond to the nicotinamide ribose and may influence $\mathrm{NAD}^{+}$binding.

Paithankar et al. reported that the closure of the substrate-binding loop was induced by cosubstrate binding in Pseudomonas putida HBDH (PpHBDH) (13). In 3a-hydroxysteroid dehydrogenase, the loop-helix transition was induced by coenzyme binding (29). To clarify the mechanism underlying the conformational change in PfHBDH, we used a highly purified preparation of the wild-type HBDH, which has been extensively dialyzed against an excess volume of $10 \mathrm{mM}$ Tris- $\mathrm{HCl}$ buffer ( $\mathrm{pH}$ 7.5). Crystals of the $\mathrm{HBDH}-\mathrm{NAD}^{+}$binary complex were made in the presence of $4 \mathrm{mM} \mathrm{NAD}^{+}$. As shown in Figure $1 \mathrm{~b}$, chain $\mathrm{A}$ was found to be a closed form. The other chains $(\mathrm{B}, \mathrm{C}$, and $\mathrm{D})$ remained in the open form. Importantly, $\mathrm{NAD}^{+}$molecules were identified in all four chains with almost the same conformation. Similar patterns were observed for the crystals made in the presence of $\mathrm{CaCl}_{2}$ instead of $\mathrm{MgCl}_{2}$. Crystals of $\mathrm{PpHBDH}$ were 
prepared in the presence of $\mathrm{NAD}^{+}$, acetoacetate, and $\mathrm{CaCl}_{2}$. The closed conformation observed in the PpHBDH-NAD ${ }^{+}$complex might have been mediated by the acetoacetate in the crystallization buffer. The closed conformation was also described for the structure of AfHBDH, which has been determined recently by Hoque et al (14). They assigned the acetate ion in the crystal structure and constructed a substrate-bound model from the acetate complex. These observations suggest that the $\mathrm{NAD}^{+}$binding makes $\mathrm{HBDH}$ competent to adopt a closed form but it is not sufficient for shifting the equilibrium from an open to a closed form. In contrast, all 4 chains of the HBDH-NAD ${ }^{+}-\mathrm{L}-3-\mathrm{HB}$ complex had a closed form. Comparable values between $K_{\mathrm{m}}$ for $\mathrm{L}-3-\mathrm{HB}$ and $K_{\mathrm{i}}$ for $\mathrm{L}-3-\mathrm{HB}$ are consistent with the similar interactions of carboxyl-, 3-methyl-, and 3-hydroxyl groups with the enzyme. These observations indicate that the substrate/inhibitor binding is required for the stable ternary complex formation.

In many of the crystal structures of SDR enzymes, the substrate-binding loop region is ordered in the presence of cofactor but is disordered in its absence (7). The disordered structure of the substrate-binding loop in the crystal of the PfHBDH-NAD ${ }^{+}$-inhibitor (PDB code: $1 \mathrm{X} 1 \mathrm{~T}$ ) was caused by the difference in the structures between the substrate and the inhibitor. We hypothesized that the disorder was 
caused by a steric hindrance between a methyl group of a cacodylate and the moving substrate-binding loop. The structures of HBDH-cacodylate binary complex (PDB code: $1 \mathrm{WMB}$ ) and HBDH-NAD ${ }^{+}$-cacodylate ternary complex were superimposed onto that of the HBDH-NAD ${ }^{+}-\mathrm{L}-3-\mathrm{HB}$ ternary complex, and the active site of the HBDH-NAD ${ }^{+}$-L-3-HB is shown along with the cacodylate anions in Figure 5. Unexpectedly, the cacodylate molecule of $1 \mathrm{X} 1 \mathrm{~T}$ can be placed without steric hindrance in the space where the L-3-HB is bound. This is possible because the enzyme has some allowance, as already described. L-threonine can be bound to the active site for the same reason, but the binding is weak because of the presence of the hydrophilic amino group facing the hydrophobic tryptophan residue. The major difference between cacodylate and hydroxybutyrate must be their negative charges. The carboxyl group can form a hydrogen bond with Gln196 on the substrate-binding loop and stabilize the closed conformation of the ternary complex. The negative charge on the cacodylate anion, however, cannot interact with Gln196, leading to a disorder of the substrate-binding loop. Substitution for Gln196 drastically decreased activity, mainly due to the large increase in the $K_{\mathrm{m}}$ value for D-3-HB. Compatible with these experimental data, Gln196 is a critical residue not only for substrate binding but also for the stabilization of the closed conformation. 
The substrate-binding loop moves as a rigid body upon substrate binding. We reported Thr190 and Leu215 functioned as hinge for the open-closed conformational change because these residues significantly changed the conformation between enzyme-cacodylate complex (open form) and enzyme-NAD ${ }^{+}$-cacodylate complex, of which substrate-binding loop was disordered (5). In this study, Arg189-Leu192 and Leu215-Ser217 appear to function as hinges for the conformational change, and a center of the hinge regions was Thr190 and Leu216, respectively. The site-directed mutagenesis of Thr190 confirmed the importance of the hydroxyl group. The hydroxyl group of Thr190 interacts with the phosphate and carboxamide groups of $\mathrm{NAD}^{+}$. No direct contact is observed between Thr190 and the substrate/inhibitor. As shown in Table 4, T190S substitution affected only $K_{\mathrm{m}}$, and $k_{\text {cat }}$ was even slightly increased, indicating that the Thr190 contributed significantly to substrate binding. This must be due to the interaction between Gln196 and the substrate. The closed conformation was confirmed in the crystal structure of the T190S enzyme-NAD ${ }^{+}$-L-3-HB complex. The methyl group of the Thr190 residue may be involved in the stability of the closed form. Similarly, Leu215 is also important for the substrate/inhibitor-induced conformational change. Since Leu215 has no direct interaction with 
$\mathrm{NAD}^{+} / \mathrm{NADH}, K_{\mathrm{m}}$ values toward the cofactor were not significantly affected. The increase in $K_{\mathrm{m}}$ values toward D-3-HB may be a result of the destabilization of the closed conformation. The isopropyl side chain may be sufficient for binding the substrates and catalysis of the reaction in association with Trp187. The small methyl side chain in L215A mutant, however, may not fully support the binding of the substrate and catalysis. We are now trying to crystallize the Leu215 mutants to clarify the role of Leu215 residue.

In conclusion, we have determined the crystal structure of the closed complex induced by L-3-HB, an enantiomeric competitive inhibitor, and clarified the importance of the interaction between the hydroxyl groups of the substrate/inhibitor and the active site tyrosine. To our knowledge, this may be the first crystal structure of the SDR family to have the enantiomeric competitive inhibitor-induced closed form, and should provide a good model to investigate the open-closed conformational change of the family. By using a pure enzyme preparation, which was free from substrate analogs, the crystal structure of the PfHBDH-NAD ${ }^{+}$binary complex was also determined, and the open conformation predominated. The hinge regions, Thr190 and Leu215, are important for the conformational change upon substrate binding. The cofactor binding may trigger the conformational change of the substrate-binding loop and make the enzyme 
competent for the closed form; however, this is not sufficient for the stable formation of the ternary complex in the closed conformation.

\section{ACKNOWLEDGEMENTS}

This work was supported in part by the Grant-in-Aid for Scientific Research from Japan Society for the

Promotion of Science (JSPS). 


\section{REFERENCES}

1. Laffel, L. (1999) Ketone bodies: a review of physiology, pathophysiology and application of monitoring to diabetes. Diabetes Metab. Res. Rev. 15, 412-426

2. Marks, A. R., McIntyre, J. O., Duncan, T. M., Erdjument-Bromage, H., Tempst, P. \& Fleischer, S. (1992) Molecular cloning and characterization of (R)-3-hydroxybutyrate dehydrogenase from human heart.

J. Biol. Chem. 267, 15459-15463

3. Kruger, K., Lang, G., Weidner, T. \& Engel, A. M. (1999) Cloning and functional expression of the D- $\beta$-hydroxybutyrate dehydrogenase gene of Rhodobacter sp. DSMZ 12077. Appl. Microbiol. Biotechnol. 52, 666-669

4. Uno, S., Takehiro, O., Tabata, R., and Ozawa, K. (1995) Enzymatic method for determining ketone body ratio in arterial blood. Clin. Chem. 41, 1745-1750

5. Ito, K., Nakajima, Y., Ichihara, E., Ogawa, K., Katayama, N., Nakahima, K., and Yoshimoto, T. (2006) D-3-Hydroxybutyrate dehydrogenase from Pseudomonas fragi: molecular cloning of the enzyme gene and crystal structure of the enzyme. J. Mol. Biol. 355, 722-733 
6. Oppermann, U., Filling, C., Hult, M., Shafqat, N., Wu, X., Lindh, M., Shafqat, J., Nordling, E., Kallberg, Y., Persson, B., and Jornvall, H. (2003) Short-chain dehydrogenases/reductases (SDR): the 2002 update. Chem. Biol. Interact. 143-144, 247-253

7. Tanaka, N., Nonaka, T., Nakamura, K. T., and Hara, A. (2001) SDR: structure, mechanism of action, and substrate recognition. Curr. Org. Chem. 5, 89-111

8. Persson, B., Kallberg, Y., Oppermann, U., and Jörnvall, H. (2003) Coenzyme-based functional assignments of short-chain dehydrogenase/reductases (SDRs) Chem. Biol. Interact. 143-144, $271-278$

9. Filling, C., Berndt, K.D., Benach, J., Knapp, S., Prozorovski, T., Nordling, E., Ladenstein, R., Jörnvall, H., and Oppermann, U. (2002) Critical residues for structure and catalysis in short-chain dehydrogenases/reductases. J. Biol. Chem. 277, 25677-25684

10. Ghosh, D., Weeks, C.M., Grochulski, P., Duax, W.L., Erman, M., Rimsay, R.L., and Orr, J.C. (1991) Three-dimensional structure of holo $3 \alpha, 20 \beta$-hydroxysteroid dehydrogenase: a member of a short-chain dehydrogenase family. Proc. Natl. Acad. Sci. USA 88, 10064-10068 
11. Tanaka, N., Nonaka, T., Tanabe, T., Yoshimoto, T., Tsuru, D. and Mitsui, Y. (1996) Crystal structures of the binary and ternary complexes of $7 \alpha$-hydroxysteroid dehydrogenase from Escherichia coli. Biochemistry 35, 7715-7730

12. Guo, K., Lukacik, P., Papagrigoriou, E., Meier, M., Lee, W.H., Adamski, J., and Oppermann, U. (2006) Characterization of human DHRS6, an orphan short chain dehydrogenase/reductase enzyme: a novel, cytosolic type 2 R-beta-hydroxybutyrate dehydrogenase. J. Biol. Chem. 281, 10291-10297

13. Paithankar, K.S., Feller, C., Kuettner, E.B., Keim, A., Grunow, M., and Sträter, N. (2007) Cosubstrate-induced dynamics of D-3-hydroxybutyrate dehydrogenase from Pseudomonas putida.

FEBS J. 274, 5767-5779

14. Hoque, M.M., Shimizu, S., Hossain, M.T., Yamamoto, T., Imamura, S., Suzuki, K., Tsunoda, M., Amano, H., Sekiguchi, T., and Takenaka, A. (2008) The structures of Alcaligenes faecalis D-3-hydroxybutyrate dehydrogenase before and after $\mathrm{NAD}^{+}$and acetate binding suggest a dynamical reaction mechanism as a member of the SDR family. Acta Crystallogr D Biol Crystallogr.

64, 496-505 
15. Ke, S.H., and Madison, E.L. (1997) Rapid and efficient site-directed mutagenesis by single-tube 'megaprimer' PCR method. Nucleic Acids Res. 25, 3371-3372

16. Otwinowski, Z. and Minor, W. (1997) Processing of X-ray diffraction data collected in oscillation mode.

Methods Enzymol. 276, 307-326

17. Nakajima, Y., Ito, K., Ichihara, E., Ogawa, K., Egawa, T., Xu, Y., and Yoshimoto, T. (2005) Crystallization and preliminary X-ray characterization of D-3-hydroxybutyrate dehydrogenase from Pseudomonas fragi. Acta Crystallogr. Sect. F Struct. Biol. Cryst. Commum. 61, 36-38

18. Vagin, A. and Teplyakov, A. (1997) MOLREP: an automated program for molecular replacement. J. Appl. Cryst. 30, 1022-1025

19. Collaborative Computational Project, Number 4 (1994) The CCP4 suite: programs for protein crystallography. Acta Crystallogr. D Biol. Crystallogr. 50, 760-763

20. Brunger, A.T., Adams, P.D., Clore, G.M., Delano, W.L., Gros, P., Grosse-Kunstleve, R.W., Jiang, J.-S., Kuszewski, J., Nilges, N., Pannu, N.S., Read, R.J., Rice, L.M., Simonson, T., and Warren, G.L. (1998) Crystallography \& NMR System: A new software suite for macromolecular structure 
determination. Acta Crystallogr. D Biol. Crystallogr. 54, 905-921

21. Emsley, P. and Cowtan, K. (2004) Coot: Model-building tools for molecular graphics. Acta Crystallogr D Biol. Crystallogr. 60, 2126-2132

22. Murshudov, G.N., Vagin, A.A., and Dodson, E.J. (1996) Refinement of macromolecular structures by the maximum-likelihood method. Acta Crystallogr. D Biol. Crystallogr. 53, 240-255

23. Laskowski, R.A., MacArthur, M.W., Moss, D.S. and Thornton, J.M. (1993) PROCHECK: a program to check the stereochemical quality of protein structures. J. Appl. Crystallogr. 26, 283-291.

24. Kraulis, P.J. (1991) MOLSCRIPT: a program to produce both detailed and schematic plots of protein structures. J. Appl. Crystallogr. 24, 946-950

25. Fenn, T.D., Ringe, D. and Petsko, G.A. (2003) POVScript+: a program for model and data visualization using persistence of vision ray-tracing. J. Appl. Crystallogr. 36, 944-947

26. Merritt, E.A. and Murphy, M.E.P. (1994) Raster3D Version 2.0: a program for photorealistic molecular graphics. Acta Crystallogr. D Biol. Crystallogr. 50, 869-873

27. Wallace, A.C., Laskowski, R.A., \& Thornton, J.M. (1995) LIGPLOT: A program to generate schematic 
diagrams of protein-ligand interactions. Prot. Eng. 8, 127-134

28. Feller, C., Günther, R., Hofmann, H.J., Grunow, M. (2006) Molecular basis of substrate recognition in D-3-hydroxybutyrate dehydrogenase from Pseudomonas putida. Chembiochem. 7,1410-1418

29. Nakamura, S., Oda, M., Kataoka, S., Ueda, S., Uchiyama, S., Yoshida, T., Kobayashi, Y., and Ohkubo, T.

(2006) Apo- and holo-structures of 3a-hydroxysteroid dehydrogenase from Pseudomonas sp.

B-0831: Loop-helix transition induced by coenzyme binding. J. Biol. Chem. 281, 31876-31884 
Table 1. Mutagenic and flanking primers

\begin{tabular}{|c|c|c|c|c|c|}
\hline \multirow[t]{2}{*}{ Mutation } & \multirow[t]{2}{*}{ Nucleotide sequence } & \multicolumn{2}{|c|}{ Flanking primers } & \multicolumn{2}{|c|}{ Restriction sites } \\
\hline & & up & down & up & down \\
\hline Q94A & 5'-CGCCGGCATCGCGCACACCGCGCTG-3' & ATG15 & $17 \mathrm{a}$ & NcoI & BglII \\
\hline H144A & 5'-CGCCTCGGCGGCCGGCCTGGTGGC-3' & $19 \mathrm{~s}$ & $17 \mathrm{a}$ & NcoI & BglII \\
\hline $\mathrm{K} 152 \mathrm{~A}$ & 5'-GGCCAACGCGTCGGCCTACG-3' & $19 \mathrm{~s}$ & $17 \mathrm{a}$ & NcoI & BglII \\
\hline $\mathrm{K} 152 \mathrm{R}$ & 5'-CGGCCAACCGGTCGGCCTACGTCGC-3' & $19 \mathrm{~s}$ & $17 \mathrm{a}$ & NcoI & BglII \\
\hline K152Q & 5'-CGGCCAACCAGTCGGCCTACGTCGC-3' & $19 \mathrm{~s}$ & $17 \mathrm{a}$ & NcoI & BglII \\
\hline $\mathrm{K} 152 \mathrm{E}$ & 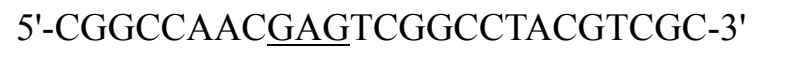 & $19 \mathrm{~s}$ & $17 \mathrm{a}$ & NcoI & BglII \\
\hline Y155F & 5'-GGCCAACAAGTCGGCC $\underline{T T C G T C G C C G-3 ' ~}$ & $19 \mathrm{~s}$ & $17 \mathrm{a}$ & NcoI & BglII \\
\hline W187A & 5'-CTGCCCGGGCGCGGTGCGCACTC-3' & $19 \mathrm{~s}$ & $17 \mathrm{a}$ & BstEII & BglII \\
\hline W187F & 5'-CTGCCCGGGCTTCGTGCGCACTC-3' & $19 \mathrm{~s}$ & $17 \mathrm{a}$ & BstEII & BglII \\
\hline W187Y & 5'-CTGCCCGGGCTACGTGCGCACTC-3' & $19 \mathrm{~s}$ & $17 \mathrm{a}$ & BstEII & BglII \\
\hline W187T & 5'-CTGCCCGGGC $\underline{A C G G T G C G C A C T C-3 ' ~}$ & $19 \mathrm{~s}$ & $17 \mathrm{a}$ & BstEII & BglII \\
\hline T190A & 5'-GGTGCGCGCTCCGCTGGTCG-3' & $19 \mathrm{~s}$ & $17 \mathrm{a}$ & NcoI & BglII \\
\hline T190S & 5'-GGGTGCGCTCTCCGCTGGTCG-3' & $19 \mathrm{~s}$ & $17 \mathrm{a}$ & NcoI & BglII \\
\hline T190C & 5'-GGGTGCGCTGTCCGCTGGTCG-3' & $19 \mathrm{~s}$ & $17 \mathrm{a}$ & NcoI & BglII \\
\hline Q196A & 5'-CTGGTCGAAAAGGCGATCTCGGCGC-3' & $19 \mathrm{~s}$ & $17 \mathrm{a}$ & NcoI & EspI \\
\hline Q196N & 5'-CTGGTCGAAAAGAACATCTCGGCGC-3' & $19 \mathrm{~s}$ & $17 \mathrm{a}$ & NcoI & EspI \\
\hline Q196E & 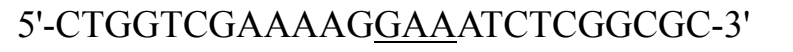 & $19 \mathrm{~s}$ & $17 \mathrm{a}$ & NcoI & EspI \\
\hline L215A & 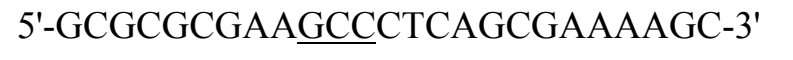 & $19 \mathrm{~s}$ & HIII-T7 & NcoI & SacII \\
\hline L215V & 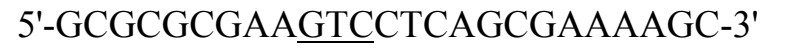 & $19 \mathrm{~s}$ & HIII-T7 & NcoI & SacII \\
\hline W257A & 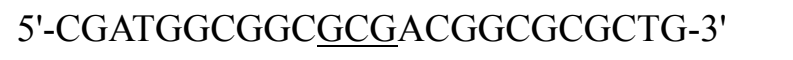 & $19 \mathrm{~s}$ & HIII-T7 & NcoI & SacII \\
\hline W257F & 5'-CGATGGCGGCTTCACGGCGCGCTG-3' & $19 \mathrm{~s}$ & HIII-T7 & NcoI & SacII \\
\hline W257Y & 5'-CGATGGCGGCTACACGGCGCGCTG-3' & $19 \mathrm{~s}$ & HIII-T7 & NcoI & SacII \\
\hline \multicolumn{6}{|c|}{ Flanking primers } \\
\hline $19 \mathrm{~s}$ & \multicolumn{5}{|l|}{ 5'-GCACACCGCGCTGATCGAGGAC-3' } \\
\hline $17 \mathrm{a}$ & \multicolumn{5}{|l|}{ 5'-CGAATTGCAGCGACGGCTGCT-3' } \\
\hline ATG15 & \multicolumn{5}{|l|}{ 5'-CCCGAATTCATGCTCAAAGGAAAAGTCGC-3' } \\
\hline HIII-T7 & \multicolumn{5}{|l|}{ 5'-GCCCAAGCTTTGTAATACGACTCACTATAG-3' } \\
\hline
\end{tabular}


Table 2. Data collection and refinement statistics

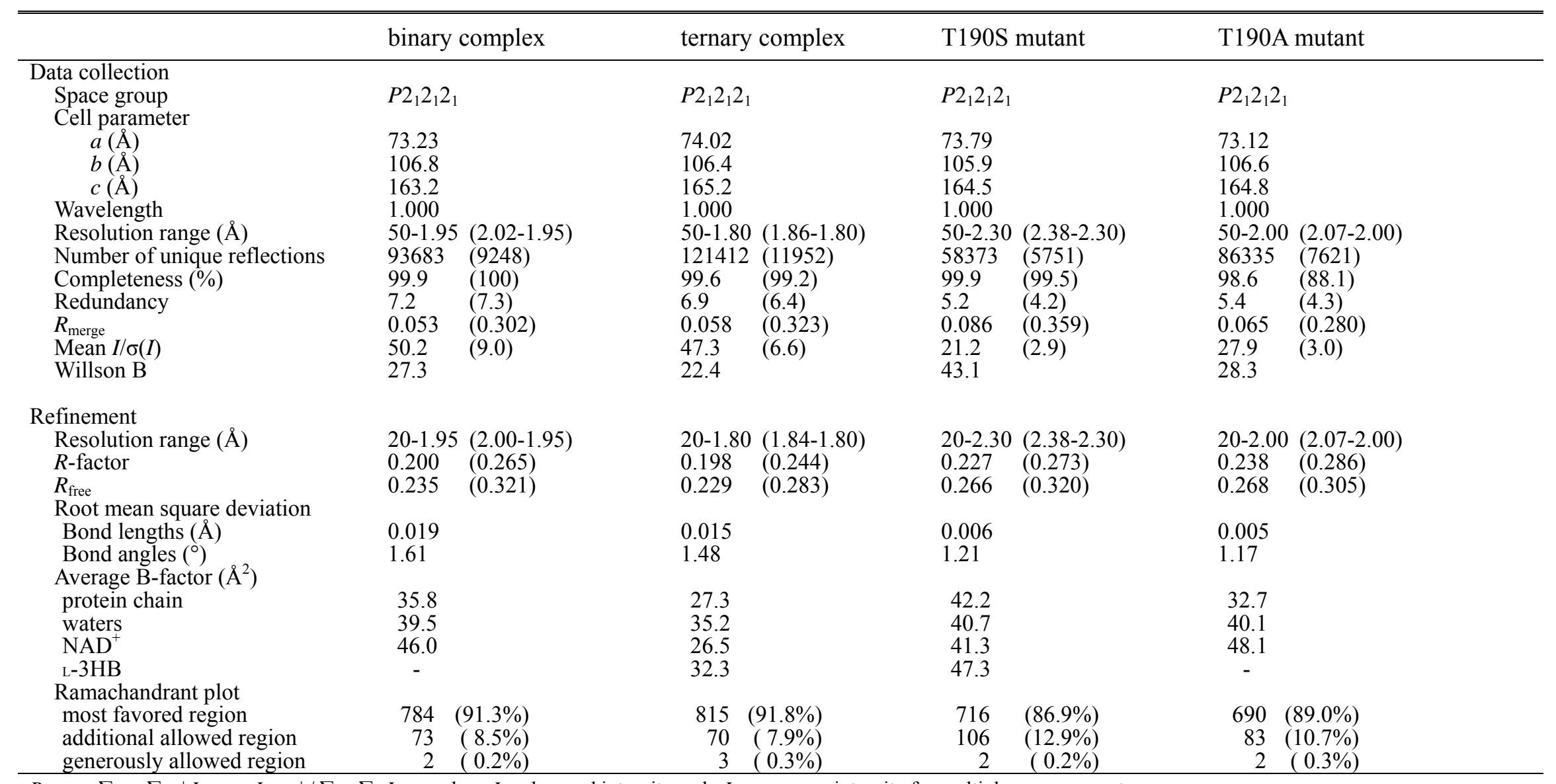

$R_{\text {merge }}=\sum_{\mathrm{hkl}} \sum_{\mathrm{i}} \mid I_{\mathrm{hk}, \mathrm{i}}-\left\langle I_{\mathrm{hkl}}>\right| / \sum_{\mathrm{hkl}} \sum_{\mathrm{i}} I_{\mathrm{hkl}, \mathrm{i}}$, where $I=$ observed intensity and $\langle\mathrm{I}\rangle=$ average intensity for multiple measurements.

Values in parentheses refer to the last resolution shell 
Table 3. Kinetic parameters of various HBDH mutants for D-3-hydroxybutyrate.

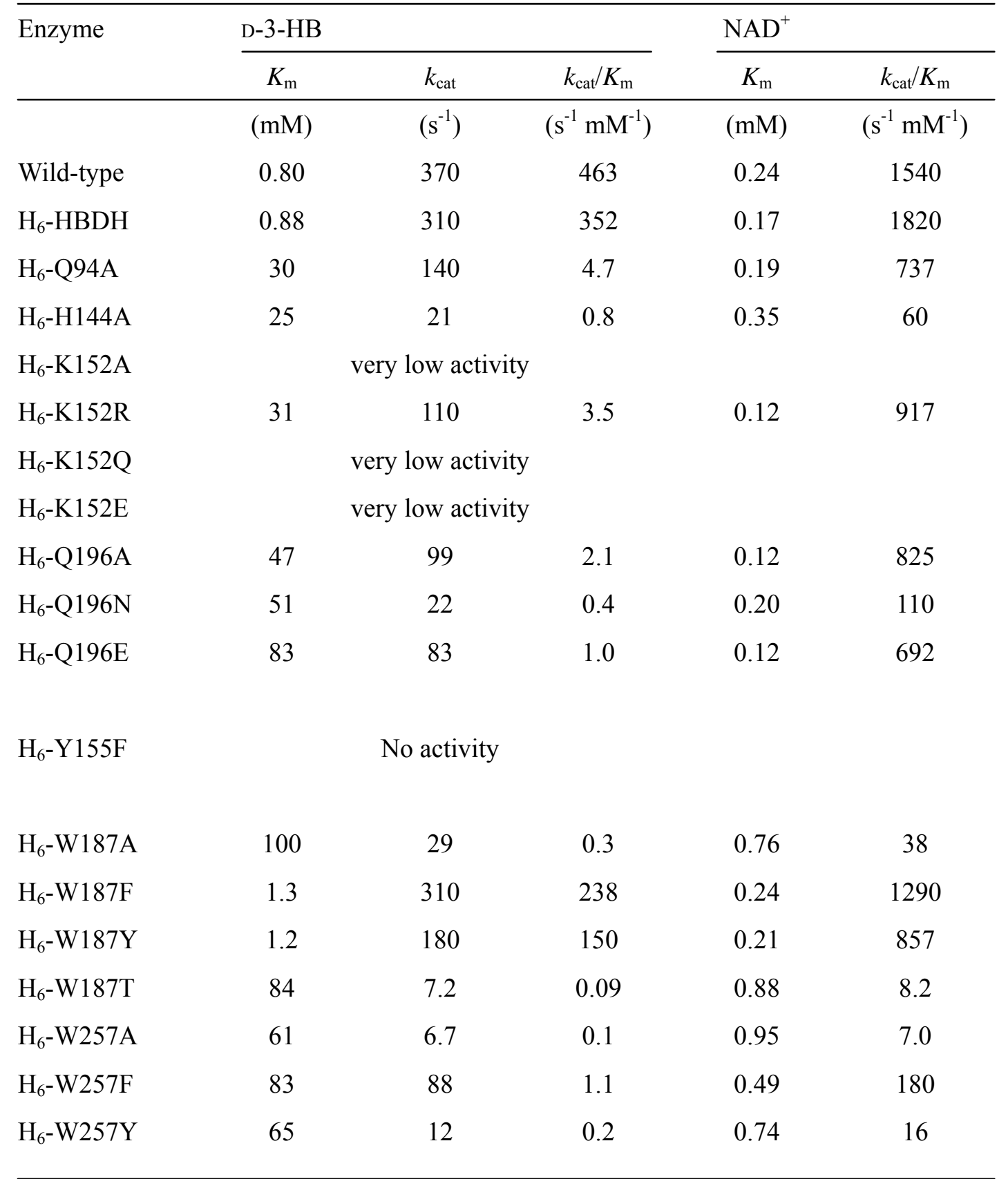


Table 4. Kinetic parameters of Thr190 and Leu215 mutants

\begin{tabular}{|c|c|c|c|c|c|c|c|c|c|c|}
\hline \multirow[t]{2}{*}{ Enzyme } & \multicolumn{3}{|c|}{ D-3-HB } & \multicolumn{2}{|l|}{$\mathrm{NAD}^{+}$} & \multicolumn{3}{|c|}{ acetoacetate } & \multicolumn{2}{|c|}{$\mathrm{NADH}$} \\
\hline & $K_{\mathrm{m}}$ & $k_{\text {cat }}$ & $k_{\mathrm{cat}} / K_{\mathrm{m}}$ & $K_{\mathrm{m}}$ & $k_{\mathrm{cat}} / K_{\mathrm{m}}$ & $K_{\mathrm{m}}$ & $k_{\text {cat }}$ & $k_{\mathrm{cat}} / K_{\mathrm{m}}$ & $K_{\mathrm{m}}$ & $k_{\mathrm{cat}} / K_{\mathrm{m}}$ \\
\hline & $(\mathrm{mM})$ & $\left(\mathrm{s}^{-1}\right)$ & $\left(\mathrm{s}^{-1} \mathrm{mM}^{-1}\right)$ & $(\mathrm{mM})$ & $\left(\mathrm{s}^{-1} \mathrm{mM}^{-1}\right)$ & $(\mathrm{mM})$ & $\left(\mathrm{s}^{-1}\right)$ & $\left(\mathrm{s}^{-1} \mathrm{mM}^{-1}\right)$ & $(\mathrm{mM})$ & $\left(\mathrm{s}^{-1} \mathrm{mM}^{-1}\right)$ \\
\hline Wild type & 0.80 & 370 & 463 & 0.24 & 1540 & 0.37 & 120 & 324 & 0.01 & 12000 \\
\hline T190S & 3.7 & 705 & 191 & 0.71 & 993 & 1.1 & 125 & 114 & 0.02 & 6250 \\
\hline T190A & 25 & 22 & 0.9 & 2.2 & 10 & 32 & 4.5 & 0.14 & 0.10 & 45 \\
\hline T190C & 36 & 6.1 & 0.2 & 0.69 & 9 & 7.7 & 5.1 & 0.66 & 1.5 & 3.4 \\
\hline L215V & 3.4 & 435 & 128 & 0.29 & 1500 & 1.3 & 87 & 67 & 0.03 & 2900 \\
\hline L215A & 9.2 & 70 & 7.6 & 1.5 & 47 & 8.3 & 30 & 3.6 & 0.5 & 60 \\
\hline
\end{tabular}


Table 5. Conformation of the individual subunits in various complexes of the wild-type, T190S, and T190A HBDHs.

\begin{tabular}{|c|c|c|c|c|c|}
\hline \multirow[t]{2}{*}{ Enzyme } & \multirow[t]{2}{*}{ PDB code } & \multicolumn{4}{|l|}{ Subunits } \\
\hline & & A & B & $\mathrm{C}$ & $\mathrm{D}$ \\
\hline Open form & 1WMB & open & open & - & - \\
\hline $\begin{array}{l}\text { Enzyme-NAD }{ }^{+}- \\
\text {cacodylate complex }\end{array}$ & $1 \mathrm{X} 1 \mathrm{~T}$ & $\begin{array}{c}\text { closed } \\
\text { (disordered) }\end{array}$ & - & - & - \\
\hline Binary complex & $2 \mathrm{ZTV}$ & closed & $\begin{array}{c}\text { open } \\
\text { (disordered) }\end{array}$ & $\begin{array}{c}\text { open } \\
\text { (disordered) }\end{array}$ & $\begin{array}{c}\text { open } \\
\text { (disordered) }\end{array}$ \\
\hline Ternary complex & $2 \mathrm{ZTL}$ & closed & closed & closed & closed \\
\hline T190S mutant & 2ZTM & closed & $\begin{array}{c}\text { closed } \\
\text { (disordered) }\end{array}$ & $\begin{array}{c}\text { closed } \\
\text { (disordered) }\end{array}$ & $\begin{array}{c}\text { closed } \\
\text { (disordered) }\end{array}$ \\
\hline T190A mutant & 2ZTU & $\begin{array}{c}\text { open } \\
\text { (disordered) }\end{array}$ & $\begin{array}{c}\text { open } \\
\text { (disordered) }\end{array}$ & $\begin{array}{c}\text { open } \\
\text { (disordered) }\end{array}$ & $\begin{array}{c}\text { open } \\
\text { (disordered) }\end{array}$ \\
\hline
\end{tabular}

ASU contains 2 subunits and 1 subunit in $1 \mathrm{WMB}\left(P 2_{1} 2_{1} 2\right)$ and $1 \mathrm{X1T}(I 222)$, respectively. For 2ZTV, 2ZTL, 2ZTM, and 2ZTU, ASU contains 4 subunits with a space group of $P 2_{1} 2_{1} 2$. 
Table 6. Variations of main-chain torsion angles at the hinge regions between the open and closed form

\begin{tabular}{rrr}
\hline Residues & $\Delta \varphi\left(^{\circ}\right)$ & $\Delta \psi\left(^{\circ}\right)$ \\
\hline Val188 & -3.2 & -7.5 \\
Arg189 & -2.7 & -1.7 \\
Thr190 & -19.0 & 7.4 \\
Pro191 & 1.8 & 9.9 \\
Leu192 & 10.6 & -12.1 \\
Val193 & -8.9 & -2.3 \\
& & \\
Glu214 & -8.8 & 0.7 \\
Leu215 & 5.5 & -6.7 \\
Leu216 & 20.0 & -29.7 \\
Ser217 & -20.5 & 13.8 \\
Glu218 & 11.1 & -0.3 \\
\hline
\end{tabular}


Figure 1

(a)

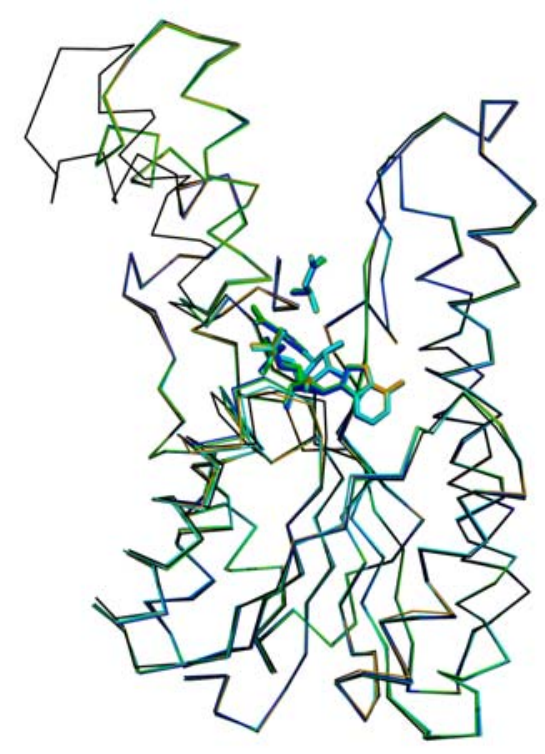

(c)

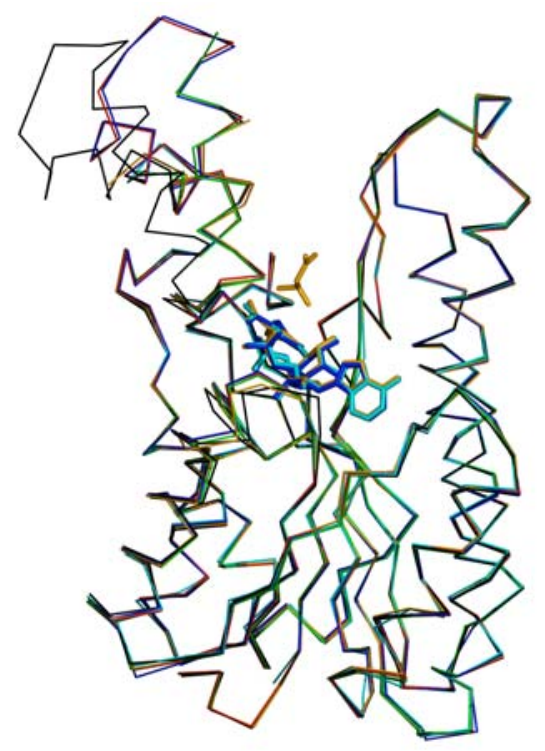

(b)

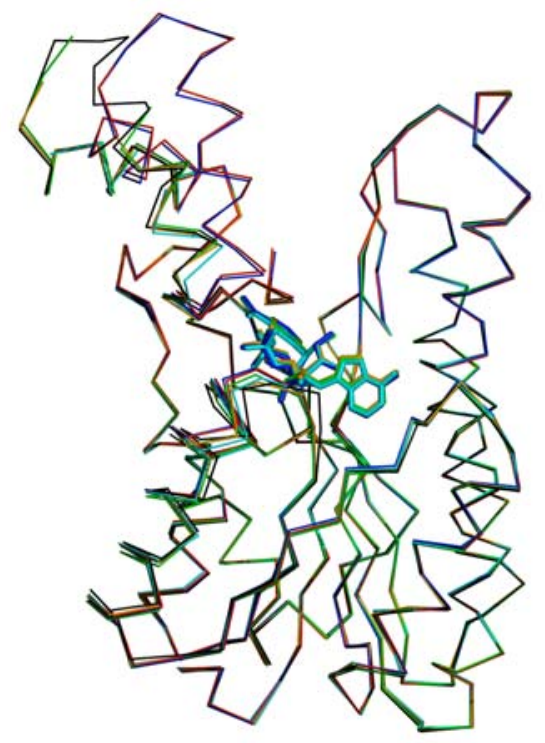

(d)

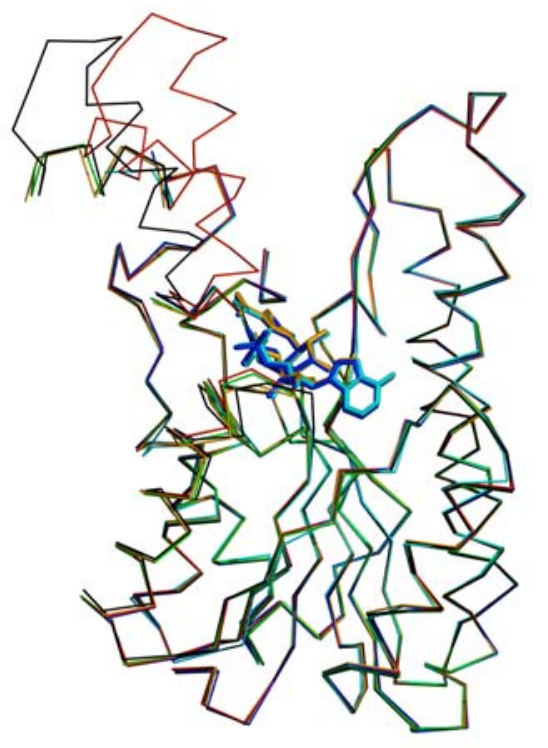


Figure 2

(a)

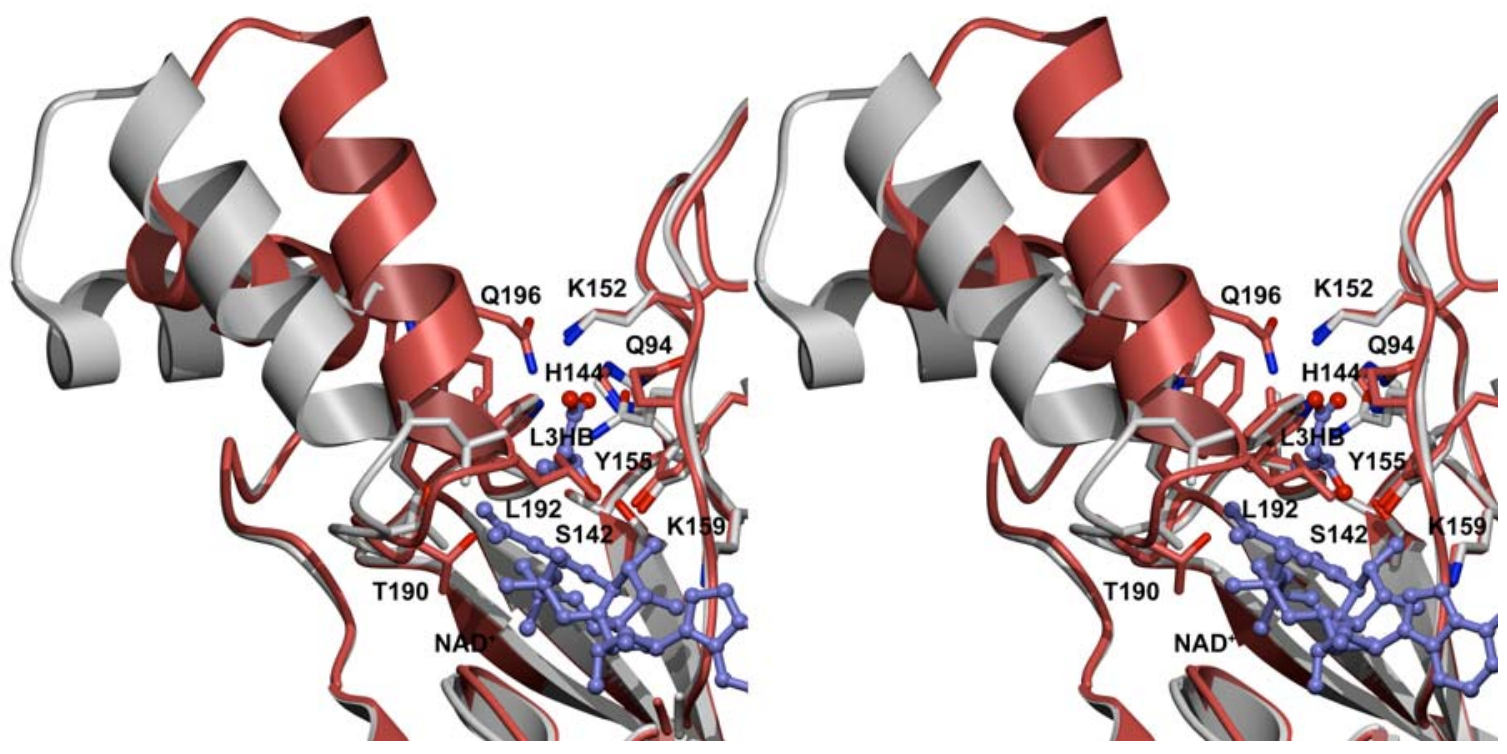

(b)

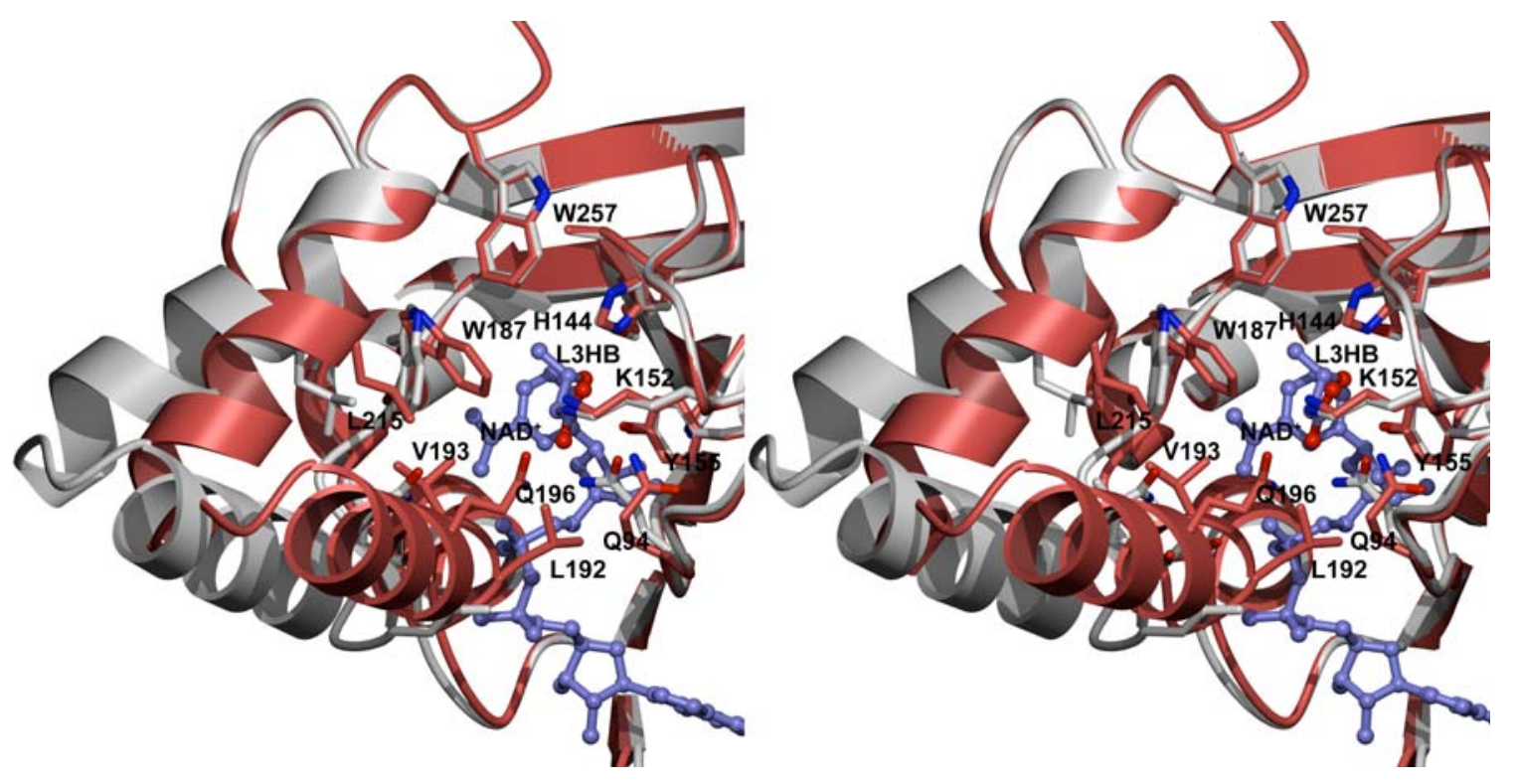


Figure 3

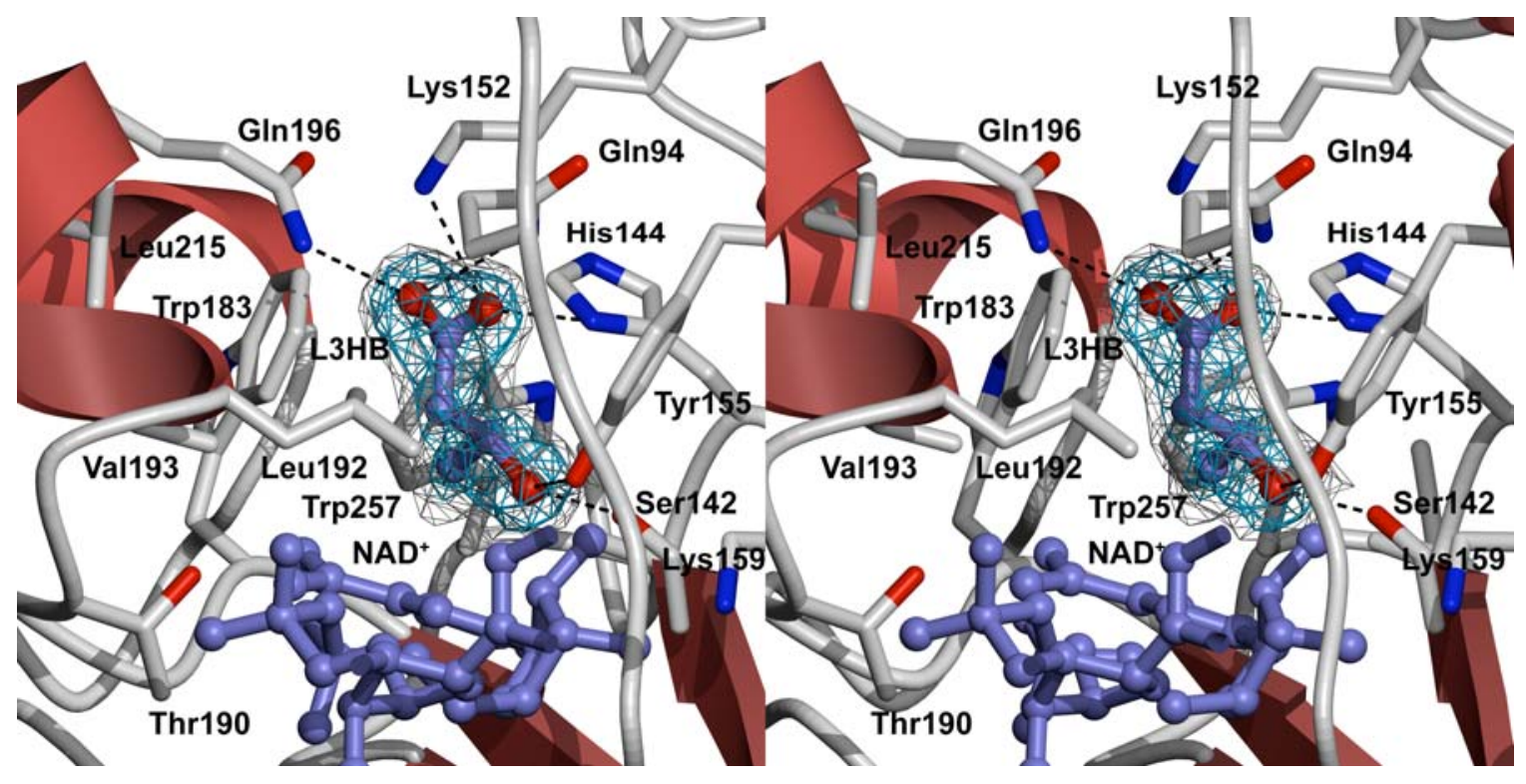


Figure 4

(a)

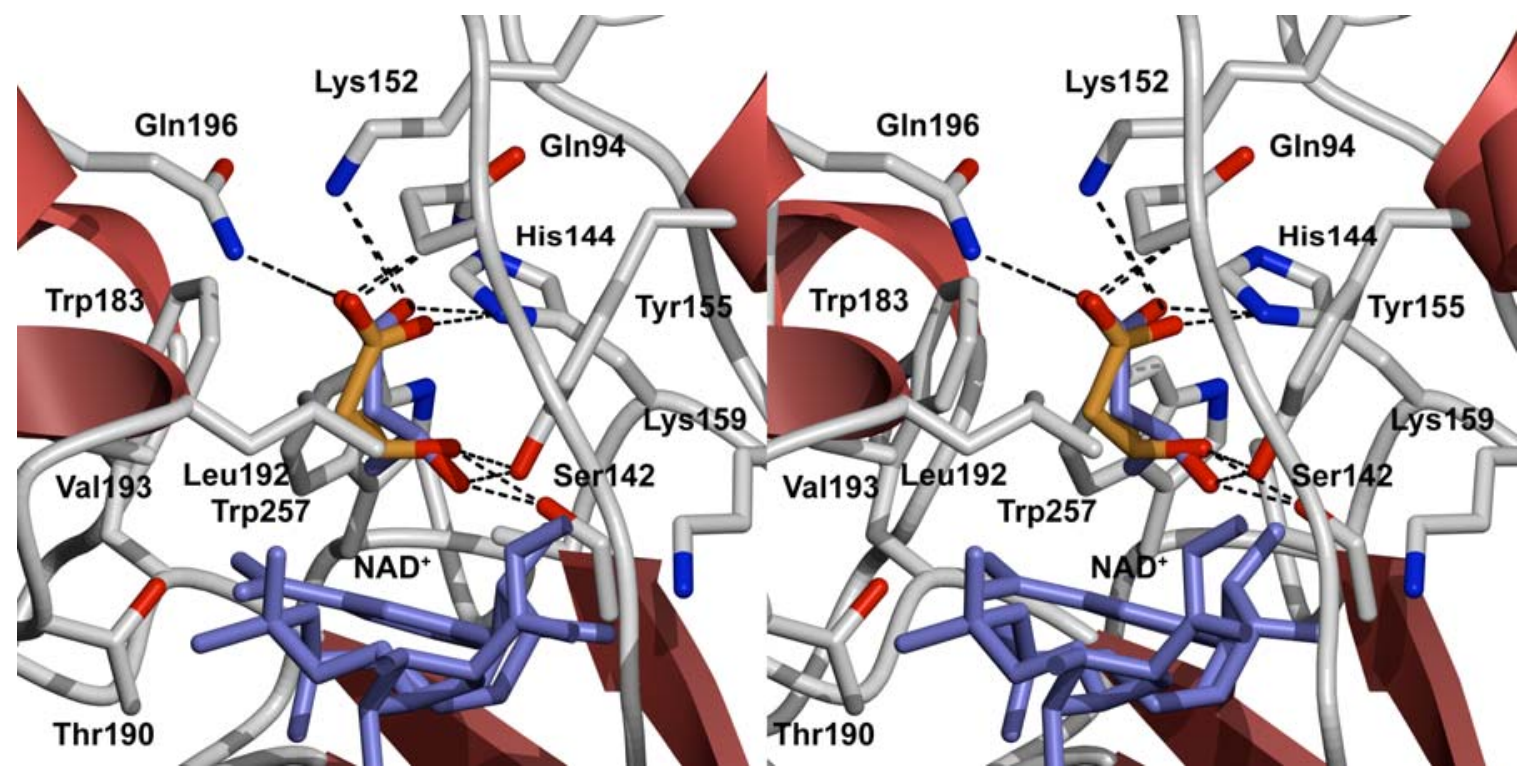

(b)

(c)
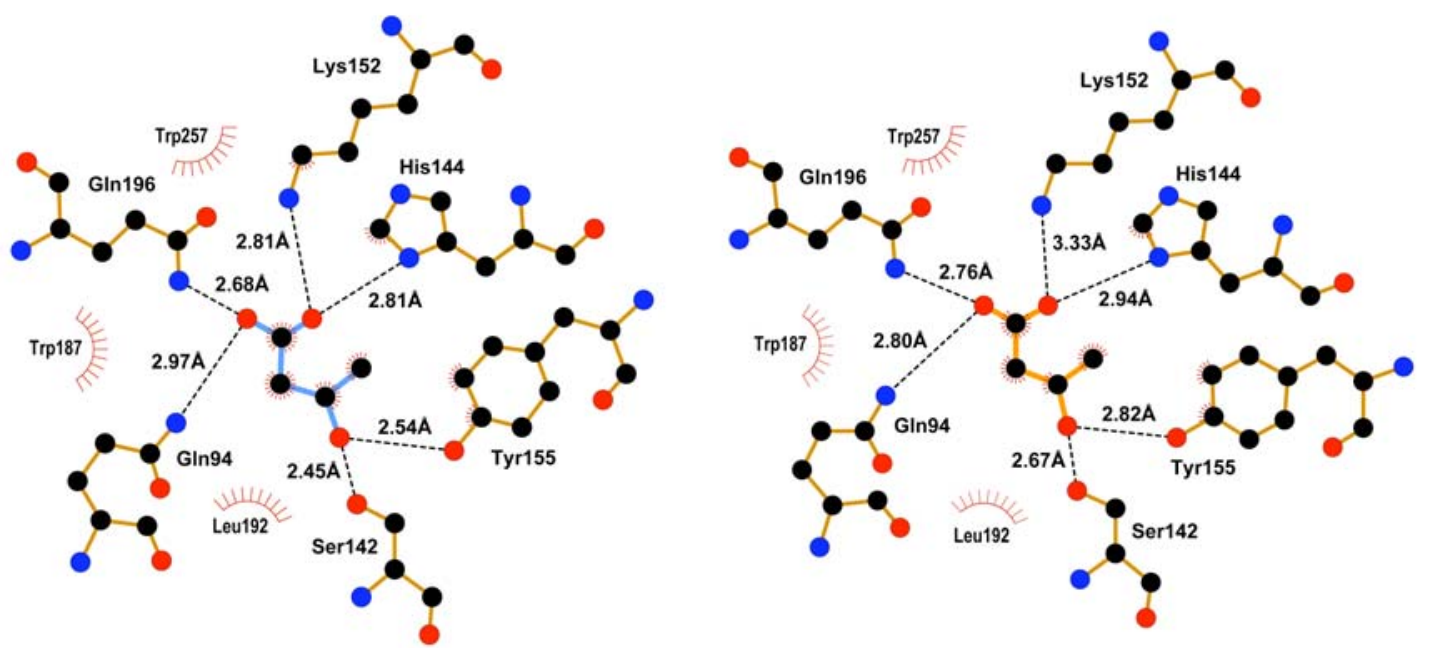
Figure 5

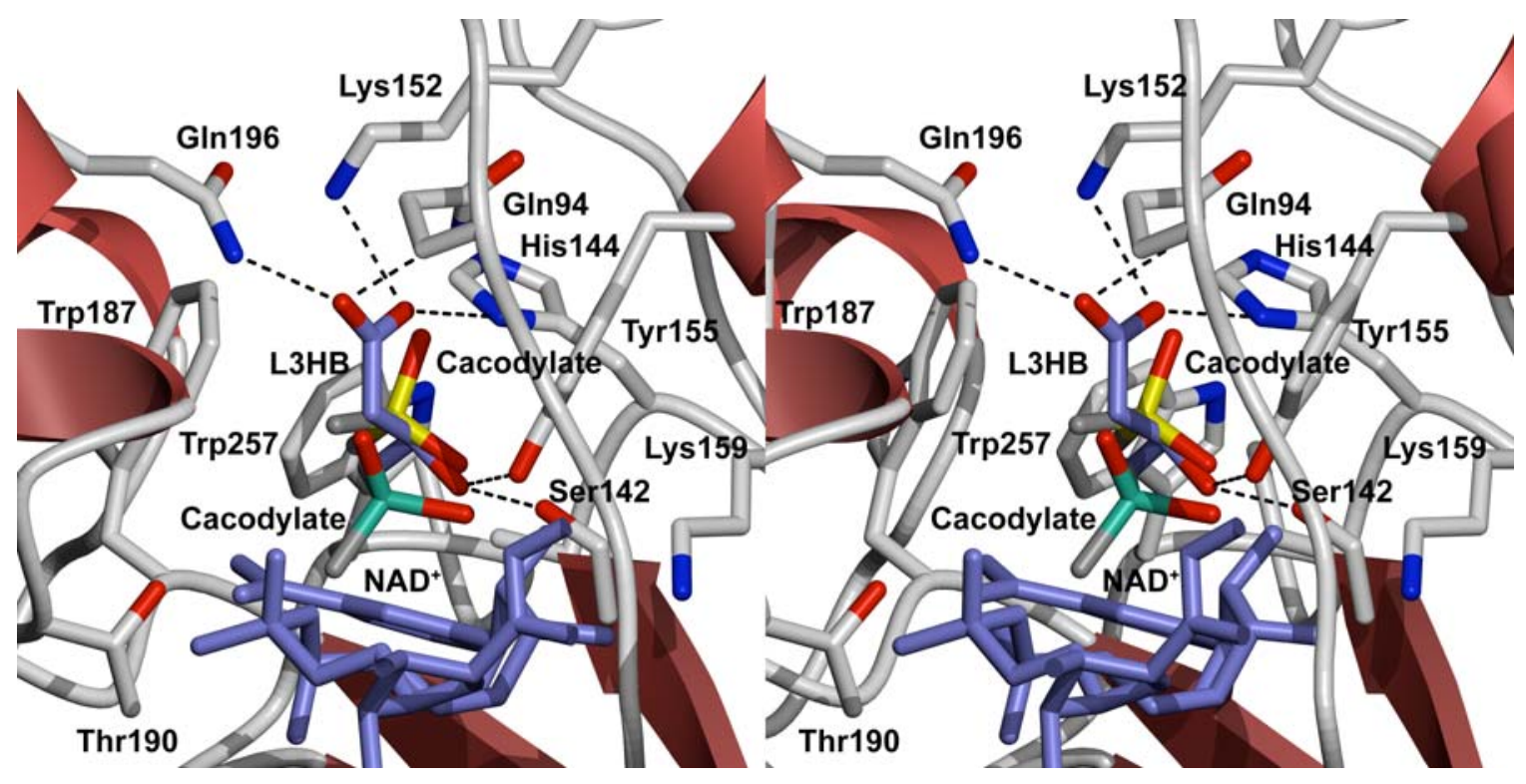

